\title{
Evaluating the Healthy Start Program: A Life Course Perspective
}

\author{
Milton Kotelchuck
}

Published online: 26 June 2010

(C) Springer Science+Business Media, LLC 2010

The Healthy Start Initiative begun in 1991 in 15 sites, now currently in 104 sites, is the largest federal program directly dedicated to reducing disparities in Maternal and Infant Health status in high risk communities. In this issue of the Maternal and Child Health Journal, two articles deriving from the most recent second national evaluation of Healthy Start Initiative are presented that characterize successfully implemented program sites from the perspectives of the local staff [1] and participants [2]. Given the life course of this program, the current evaluation reflects on Healthy Start in its "adolescence" — a young, fully operational program; no longer an "initiative" but now a legislatively mandated program. However, Healthy Start is not yet a robust, institutionalized, national "adult" program; it still reflects much local community variability and experimentation with new programmatic ideas to achieve its disparity reduction goals. These two articles, viewed from a life course perspective, describe the Healthy Start Initiative currently, place its achievements and limitations in context, and point to several new enhancements to strengthen it as it goes forward.

The Healthy Start Program's principle features are its multilevel integration of efforts to improve both the local perinatal services and systems, with extensive community participation and voice. It is implemented through nine required components; five direct service components (outreach, health education, case management, perinatal depression screening and referral, and inter-conception care) and four system components (consortium, state

\section{Kotelchuck ( $\square)$}

Department of Community Health Sciences, Boston University School of Public Health, 801 Massachusetts Avenue, Room 434, Boston, MA 02118-2605, USA

e-mail: mkotelch@bu.edu
Title V collaboration, local health systems action plan, and sustainability plan). As the second article notes [2], Healthy Start's underlying causal model suggests that local community involvement will lead to improved services and systems tailored to local cultural and linguistic needs, which will lead to increased use of appropriate health care and other services and enhanced health behaviors, which will bring about improved maternal and infant health outcomes and ultimately help eliminate racial and ethnic disparities.

The most recent national Healthy Start Program evaluation had two distinct components: a survey of all then 96 Healthy Start sites; followed by eight in depth aggregated case studies of successfully implemented sites and an associated survey of a sample of their participants. This was not a traditional "outcomes" or "health service utilization" orientated evaluation, but rather a self-conscious, management oriented evaluation, to intentionally look at its own functioning, to assess areas of strength and potential improvements - a very appropriate evaluation for this adolescent stage of a program's development. The results from the eight case studies are very consistent with the findings from the survey of the 96 sites [3], but provide greater qualitative depth and insight.

\section{Management and Program Content}

Overall, the evaluation suggests that Healthy Start is a well implemented program, that all nine required components are being actively addressed but that there is great variability in how the different sites function and have implemented the required components. This variability suggests that there may be room for improvements in program implementation and in the content of the nine required 
components. Healthy Start reflects its blended early history of broad local initiatives now balanced with more programmatic requirements. As it evolves as a national program, it may need even clearer guidelines (or specified model types) within each of its components. Specifically, the evaluations found that:

1) Healthy Start is a very well liked program-both explicitly from the clients and implicitly from the local staff (who perceive that it has numerous strengths). This reveals important political will for the program. It provides a good community and national foundation on which to further build this Program.

2) The programmatic heart of Healthy Start remains its original components: outreach, care management, and health education and community involvement (consortia). They are popular, widely perceived as successful, and their barriers relatively minor. Despite Healthy Start's evident success in reaching very vulnerable populations and engaging them in enhanced perinatal services, the local sites still noted a continuing struggle to find the hard to reach newer less traditional immigrant groups and now working mothers. Also, while Healthy Start appears to help ensure high levels of perinatal health education, several sites reported client resistance and an unwillingness to change cultural practices. Healthy Start's core has evolved, but not changed fundamentally from its earliest conception.

3) The newer service initiatives (depression screenings and inter-conception programming) appear to be widely implemented, although less so than other traditional core services, and more barriers to their implementation are noted. These newer service initiatives, however, reflect well on the Healthy Start Program-being the first national program to widely implement them. They show the adolescent-stage Programs' willingness and enthusiasm to move beyond traditional $\mathrm{MCH}$ programming into the newer evolving initiatives. The emerging male fatherhood initiatives further reflects this continued evolution. Healthy Start's implementation experiences provide valuable lessons for the country. Case managers note the high levels of mental health/substance abuse service needs among participants, and also bemoan the absence of sufficient services for them, especially those without health insurance. The inter-conception care experiences suggest client retention is a serious problem. The challenge is to encourage women to want/desire post partum care (similar to prenatal care) and make the benefits of participation more valuable than the hassle of life to get there. Attractive options, like Baby Colleges or Centering Parent Programs, might enhance participation. This evaluation has already led to a major new initiative to improve inter-conception care within Healthy Start.

4) The concept of the Healthy Start consortia continues to evolve. The evaluation suggests that there may be a confusion of roles, or concepts, differentially played out at each local site's consortia- either as a mechanism for strategic planning for systems development or as a mechanism for local community members' involvement and their health education/training. That six of the case sites had dual structures for their consortia suggests the depth of this duality. Both sectors represent "community involvement", but are in fact different, albeit overlapping, communities. Both "voices" are needed to improve a community's maternal and infant health. Perhaps Healthy Start in the future should encourage two community groupings -with different names (not consortia) - to meet these two distinct Healthy Start functions.

5) Healthy Start's other -non-consortia- "systems" components present a more mixed picture. They are less well implemented, relatively under developed, and less often perceived as important, though all sites did report they had achieved progress in coordinating or integrating their local service systems. The Local Health Systems Action Plan, in particular, seems not to have been an effective programmatic activity. Perhaps the lack of power of the Healthy Start staff to be able to implement system changes or influence major area health institutions (given its small size, small budget, and who it represents-mostly poor women of color) has diminished enthusiasm for this core component of Healthy Start. Clearly, high risk communities need enhanced systems of care. Healthy Start needs to rethink how to concretely achieve this, and better retain its balance of service and systems approaches, even given limited local site funding.

The substantial programmatic variability revealed by the current national evaluation suggests that there is much room for further implementation and programmatic improvements in the Healthy Start Program. While local community adaptation is important, the core needs of high risk communities are relatively similar, and over time with program maturity should come stronger programmatic specificity and enhanced program fidelity (e.g., a more limited range of implementation models within Healthy Start's required content areas).

\section{Healthy Start Impact}

While not an outcome study, the results of the survey of clients [2] provides important insights into the impact of 
the program. In addition to its effective engagement of highly vulnerable women into enhanced perinatal care, the Healthy Start Program has had a positive impact. Program clients were more likely to engage in positive health behaviors-more infants sleeping on their back and breastfeeding. The mothers' needs were reasonably well met, and they had been exposed to a high level of perinatal education. However, no immediate birth outcomes benefits were noted. Healthy Start participants, especially African American women, did not indicate that their birth outcomes were different than other high risk women nationally; racial disparities persisted. These results are consistent with the first national Healthy Start evaluation and the rising rates of poor birth outcome nationally, especially in minority communities. They reflect yet again on Healthy Start's conundrum, it provides improved services to the most vulnerable women, but their immediate birth outcomes don't consistently improve.

Perhaps in the early initiation of Healthy Start, the expectation was established that the Program could and should directly reduce birth outcome disparities. Healthy Start was initially announced as an initiative that would "reduce infant mortality by $50 \%$ in 5 years", reflecting the then widely held view that access to comprehensive prenatal care would improve birth outcomes. Today we know that this optimistic thinking is not accurate. $\mathrm{MCH}$ life course models suggest that the life time experiences and exposures women (and populations of women) bring to their pregnancies strongly determine their differential birth outcomes.

The MCH life course framework also suggests that the strategic role and responsibility of Healthy Start for improving birth outcomes must be reframed. Healthy Start can't do it all and can't do it alone. Currently, Healthy Start only addresses perinatal health once a pregnancy begins and then it addresses only a narrow range of themes that impact on poor birth outcomes (and fewer of its deeper longitudinal root causes). Healthy Start sites reflect the social realities for poor Americans and are limited in what they can do to address them. While Healthy Start provides critical help and "glue" in assuring receipt of needed primary health care services for high risk vulnerable pregnant and post-partum women and in educating them on the best current health promoting disease preventing maternal and infant health practices, the impact of these activities on immediate birth outcomes remains limited. However, their impact on the future health of mothers and infants may be much more important than we have thought to date. Life course models have once again focused a much stronger attention onto the prenatal environment and prenatal care for the achievement of optimal health over the life course (e.g. the Barker hypothesis). As currently conceived, Healthy Start alone will unlikely be a major source of reduction of birth outcomes or disparities in America. Healthy Start is not a silver bullet. Rather, Healthy Start must be a part of a broader, more comprehensive set of longitudinal initiatives throughout high risk communities to enhance birth outcomes and subsequent maternal and infant health. That is, Healthy Start is a necessary, but not a sufficient program, to address the perinatal impacts of racial/ethnic and social class disparities in America. Like Head Start for early education, Healthy Start is important, but it is not a cure-all and should not be expected to be.

\section{Healthy Start Evaluations}

These two evaluation articles in the Maternal and Child Health Journal demonstrate once again the importance of continuously evaluating the Healthy Start Program. Formal evaluations are critical for assessing the program as a whole (beyond simply project officer oversight, anecdote, or single site reports). The ABT Associates/Mathematica Policy Research evaluators are to be complemented for the quality and comprehensiveness of their recent efforts on the National Evaluation of Healthy Start. The eight local case-study evaluations [1] appear to be very well implemented and creatively summarized and analyzed across sites. The use of the nationally representative ECLS-B data base [2], standardized to the survey participants' characteristics, provides for a very effective and creative benchmark comparison. The Healthy Start Program, conceived of as a community-based initiative, is not easy to evaluate. It has substantial variability at the local site level and lacks readily available comparison populations, nor is randomization an option. Moreover, systems efforts, whose impacts affect participants and non-participants alike, are very difficult to assess. These two articles should help demonstrate the importance of ongoing evaluations for Healthy Start Program management and strategic planning. Reporting of key information/data from each site on an annual basis is not a sufficient substitute for a formal evaluation. MCHB should be praised for encouraging the publication of these national evaluations in peer reviewed journals.

These two evaluation reports also suggest ideas for future evaluation efforts. Future evaluations should move away from the current primarily descriptive evaluations to more performance-based evaluations. The current evaluation sampled successful implementation sites, not effective outcomes sites. They should use local variations more, not to measure their distributions, but as desirable independent factors to be assessed for their effectiveness. Evaluation goals change as programs mature. Programs in their adolescence, like Healthy Start, need implementation and programmatic component assessments and maybe limited 
outcome evaluations-but more mature programs, which Healthy Start is becoming, need more outcome oriented evaluations, and comparisons of specific models within a programmatic area. Not all local sites activities are equal.

\section{MCH Life Course Theory and Practice}

The Healthy Start Program should embrace MCH life course theory more and begin to put its ideas into practice. That is, Healthy Start could further re-align its current interventions derived from its older underlying causal model (of comprehensive prenatal care improves birth outcomes) to those emerging from MCH life course models. Philosophically, this shouldn't be problematic, as life course concepts appear compatible with most Healthy Start staff's fundamental sense of how social determinants influence health and health disparities over time. Indeed, the Healthy Start Program in part already embraces social determinant theory, though not adapted to a longer life course perspective. The challenge is how to do it; how to transform theoretical life course models into Healthy Start practice, and yet retain the strength of the current Program for vulnerable women and infants. This is the challenge for the Healthy Start staff and their communities, and for all of us in the MCH field. A few brief initial observations and suggestions.

Healthy Start could have a stronger, more conscious longitudinal orientation and programming. Healthy Start has made great strides with its inter-conception care initiative-but it could do more. Inter-conception care could be reconceptualized-not as a distinct phase or programmatic component, tied to the end of the pregnancy, but more as the beginning (or continuation) of a new women's health or parent's health initiative. There is need for more emphasis on the sequelae of pregnancy among women with chronic physical or mental health issues. Do all women with gestational diabetes have post-partum glucose tolerance testing and enhanced nutrition counseling? Healthy Start should emphasize more that inter-conception care is a critical moment for future life-course disparities reduction, since the most vulnerable women are the least likely to receive a post-partum clinical visit or to stay in post-partum care programs.

Healthy Start could further enhance continuities between prenatal and postnatal (and women's) care. Family planning and reproductive life planning should be discussed not just in the inter-conception period, but throughout the pregnancy as well. The content of interconception care could have an even greater focus on longitudinal themes, such as smoking cessation, obesity/ weight management, stress reduction, etc., and perhaps even raise new topics like financial literacy. Women's preconception health care should be offered all the time, at every health service encounter. Healthy Start's services are well positioned for life course programming; they are already using implicit longitudinal service models (interconception care, men's initiative, even depression screening and referral), and its health education topics could be further MCH life course focused.

MCH life course theory also suggests new ways to think about and enhance health systems activities, in particular, the need to assure more vertical, horizontal, and longitudinal handoffs/continuities. Vertical continuities refer to linkages within the health care system, for example from Healthy Start to primary care to specialty care depending on a women's or infants' need. Medical homes, in part, help assure vertical health care continuity. The current Healthy Start Program focuses primarily on systems of health care (vertical integration); but the new MCH life course/social determinants models emphasize a broader set of "systems" linkages (horizontal integration) is critical to assuring maternal and infant health longitudinally. Indeed the unmet needs noted by women [1] (housing, child care, and dental care) reinforce the importance of multiple health, education/development, and welfare system linkages. Maybe the Local Health Systems Action Plan should be reformatted as the Local Life Course Action Plan. And longitudinal continuity, leaving the Healthy Start Program, also needs more thought. Perhaps Healthy Start should only "discharge" women/infants when they are handed off successfully into an integrated system of care-a new inter-conception "discharge" protocol-possibly involving a medical home, participation in WIC, enrollment in Head Start, and receipt of all eligible welfare/housing benefits. Healthy Start would benefit from less categorical temporal thinking and more transitory longitudinal thinking.

Similarly, from a population-based life course perspective, Healthy Start should put additional emphasis on group/community empowerment and responsibility, and less focus on individual women's responsibility only. Healthy Start might consider more formal group centered activities, for example more Centering Pregnancy and Parenting Programs. By sharing their experiences, women may be more empowered both individually and collectively to address common community-wide, but individually experienced, health and social problems, and ultimately begin to address some of the deeper social determinants of poor maternal and infant health.

The MCH life course model emphasizes the broader causal explanations and the need for longer term investments and interventions to address disparities in maternal and infant health. The capacity of the Healthy Start Program to change the reality of poverty for vulnerable women in America is limited. It must therefore use its limited funds wisely, and not try to address all local social issues 
by itself. The MCH life course approach suggests partnering with others working on common linked social justice issues, such as Medical/Legal Partnerships or Reach Out and Read or local 0-5 Coalitions geared toward school readiness. Maternal and infant health disparities are not unrelated to other health, education, welfare, employment and housing disparities in a local community.

\section{Conclusions}

The two studies that appear in this issue, as part of the current national evaluation of the Healthy Start Program, provide a detailed snapshot of 8 Healthy Start sites, from the perspectives of their program staff and their clients. Together they reveals a program in its "adolescence", achieving its three core missions: to provide critical public health services and education for vulnerable women (e.g., closing the service/education disparities gap), to provide a mechanism to assure coordinated attention to local health systems issues, and to provide a voice for local community residents involvement. Healthy Start's nine required components are widely implemented, and Healthy Start is open to new programmatic opportunities. It is well liked by both participants and staff. However, Healthy Start is also in need of further enhancements to achieve its larger maternal and infant health disparity reduction goals. This is a good moment for new strategic thinking about the Program. First, although Healthy Start appears reasonably effective in its implementation and fidelity to Healthy Start program characteristics, the wide range of local variability suggests that there is still much room for enhancing both program implementation and the 9 currently required programmatic components. With maturity, should come greater programmatic clarity and focus. Second, the strategic orientation of Healthy Start should be revisited; its current underlying causal logic model derives from an older conception of the impact of enhanced perinatal services that may no longer be adequate to guide Healthy Start. Healthy
Start should embrace the MCH life course model, and move its programs into greater alignment with its principles. Third, and finally, formal evaluations are critically important to the enhancement of the Healthy Start program. Future evaluations should focus less on understanding/characterizing local Healthy Start implementation and more explicitly on examining the program's effectiveness to improve maternal and child health outcomes and comparing alternative practice models.

In sum, we must utilize the lessons learned from the second national evaluation to strengthen Healthy Startwhile it still has its youthful vitality to explore new ways to improve its functioning, both in terms of programmatic fidelity (implementation) and programmatic content-in order to optimize the health of mothers, infants, and families now and over their life course. The challenge of maternal and infant health disparities remains. While the Healthy Start Program alone can not solve the disparities, it remains one of the critical vehicles to address them. We must retain what is positive about the Program and help it become an even more effective Program. Healthy Start owes that to its local communities, and to the country. These two articles will help shape the Healthy Start Program's future enhancements.

\section{References}

1. Brand, A., Walker, D. K., Hargreaves, M., \& Rosenbach, M. Intermediate Outcomes, strategies, and challenges of eight healthy start projects. MCHJ. doi:10.1007/s10995-008-0421-6.

2. Rosenbach, M., O’Neil, S., Cook, B., Trebino, L., \& Walker DK. Characteristics, access, utilization, satisfaction, and outcomes of healthy start participants in 8 sites. MCHJ. doi:10.1007/s10995009-0470-1.

3. Health Resources and Services Administration, Maternal and Child Health Bureau. (2006). A profile of healthy start: findings from phase I of the evaluation 2006. Washington: Government Printing Office. 